AIAA-2004-0496

\title{
ANALYTICAL MODELING OF NATURAL CONVECTION IN CONCENTRIC SPHERICAL ENCLOSURES
}

\author{
P. Teertstra ${ }^{\dagger}$, M. M. Yovanovich ${ }^{\ddagger}$ and J. R. Culham ${ }^{\star}$ \\ Microelectronics Heat Transfer Laboratory \\ Department of Mechanical Engineering \\ University of Waterloo \\ Waterloo, Ontario, Canada N2L 3G1 \\ http://www.mhtlab.uwaterloo.ca
}

\begin{abstract}
A modeling procedure is developed for natural convection heat transfer from an isothermal heated sphere located at the center of an isothermal, cooled, sphericalshaped enclosure. The model is based on the linear superposition of conduction and convection solutions, where the convective component is determined based on a combination of two limiting cases, laminar boundary layer convection and transition flow convection. The model is validated using experimental and numerical data from the literature, including data from an experimental test program performed by the authors in a previous publication for four different enclosure configurations, $1.5 \leq d_{o} / d_{i} \leq 4.8$, over the range of Rayleigh number $10 \leq R a_{\sqrt{A_{i}}} \leq 1 \times 10^{7}$. The model accurately predicts the experimental data with an RMS difference of $2-4 \%$ and is in good agreement with the other data from the literature.
\end{abstract}

\section{Nomenclature}

$\begin{array}{ll}A & =\text { area; }\left(m^{2}\right) \\ C & =\text { coefficient } \\ \bar{C}_{c s} & =\text { Raithby and Hollands }{ }^{16} \text { coefficient } \\ d & =\text { diameter; }(m) \\ F(P r) & =\text { Prandtl number function } \\ g & =\text { gravitational acceleration; }\left(\mathrm{m} / \mathrm{s}^{2}\right) \\ G_{\mathcal{L}} & =\text { body gravity function } \\ k & =\text { thermal conductivity; }(\mathrm{W} / \mathrm{mK}) \\ k_{e} & =\text { effective thermal conductivity, } \\ \mathcal{L} & =\text { general characteristic length; }(\mathrm{m})\end{array}$

${ }^{\dagger}$ Research Assistant Professor

¥ Fellow AIAA, Distinguished Professor Emeritus

* Associate Professor, Director MHTL

Copyright (C) 2004 by the authors. Published by the American Institute of Aeronautics and Astronautics, Inc., with permission.
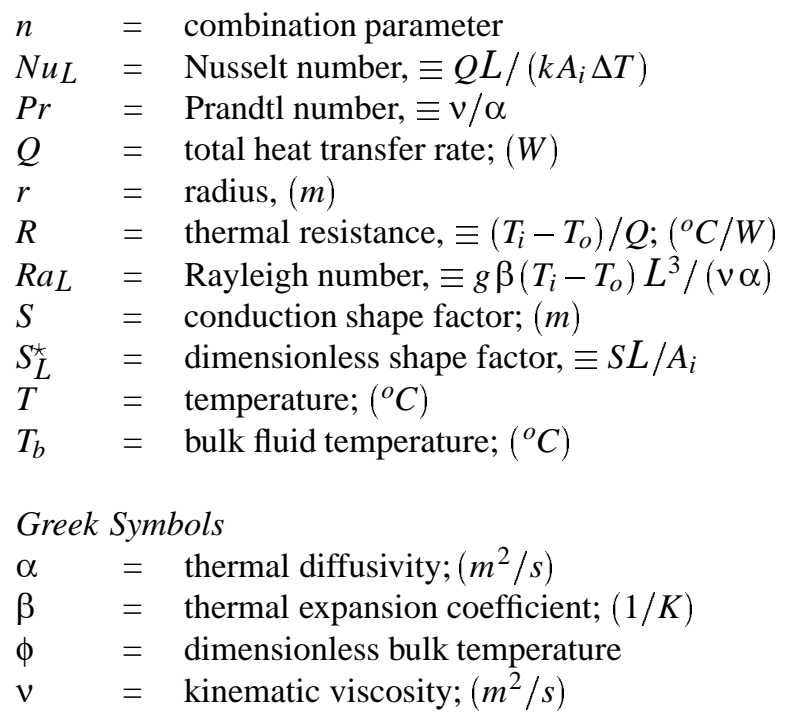

\begin{tabular}{lll}
\multicolumn{2}{l}{ Subscripts } \\
$b \quad=$ & bulk fluid \\
$\mathrm{bl}$ & $=$ & boundary layer flow \\
$\mathrm{conv}$ & $=$ & convection \\
$\mathrm{tr}$ & $=$ & transition flow \\
$i$ & $=$ & inner body \\
$o$ & $=$ & outer body \\
$L B$ & $=$ & lower bound \\
$U B$ & $=$ & upper bound
\end{tabular}

\section{Introduction}

Natural convection in enclosures has been widely studied both experimentally and analytically by a number of researchers for applications spanning a variety of disciplines, including nuclear reactor design, energy transmission and storage, solar energy and microelectronics systems. Of particular interest to the designers of sealed equipment cabinets for telecom and wireless applications is natural convection in the enclosure formed between a 


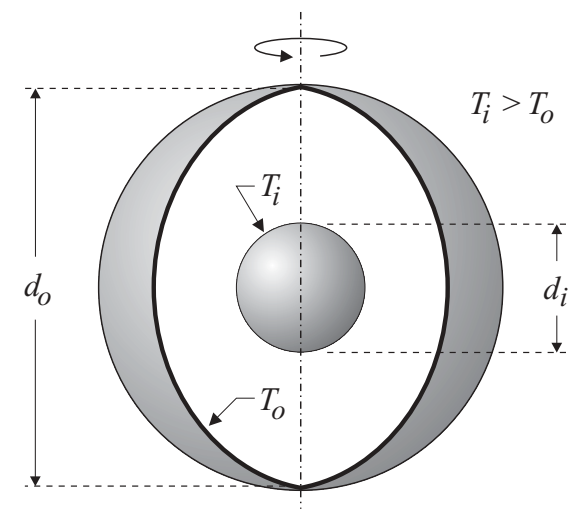

Fig. 1 Schematic of concentric spherical enclosure

heated body and its surrounding cooled enclosure. The formulation of easy-to-use design tools for the thermal analysis of these equipment provides a mechanism for quickly and accurately predicting operating temperatures and performing trade off and parametric studies, prior to more costly and time consuming CFD analysis or prototype testing.

The problem of interest in the current study involves natural convection between an isothermal heated sphere of diameter $d_{i}$ located at the center of an isothermal, cooled spherical enclosure of diameter $d_{o}$, as shown in Fig. 1. This type of enclosure is characterized by a fluid region that has clearly defined, non-intersecting inner and outer boundaries, such that heat transfer occurs between the inner and outer boundaries only. Although not indicative of the more complex geometries found in typical electronics enclosures, the models developed for the concentric spheres will form the basis for future models of more complex enclosure configurations.

Experimental and numerical data for natural convection heat transfer in the concentric spheres has been presented by a number of researchers over the past forty years. Bishop et al. ${ }^{1,2}$, Mack and Hardee ${ }^{3}$, Scanlan et al. ${ }^{4}$ and Weber et al. ${ }^{5}$ present experimental data for the concentric spherical enclosure, focusing on the high Rayleigh number, laminar boundary layer flow regime. Most of the remaining studies involve numerical simulations of the spherical enclosure, including Mack and Hardee $^{3}$, Astill et al. ${ }^{6}$, Caltagirone et al. ${ }^{7}$, Singh and Chen $^{8}$, Ingham ${ }^{9}$, Wright and Douglass ${ }^{10}$, Fujii et al. ${ }^{11}$, Garg $^{12}$, Chu and Lee ${ }^{13}$ and Chiu and Chen ${ }^{14}$. Teertstra et al. ${ }^{15}$ present experimental data for four different concentric spheres, $d_{o} / d_{i}=1.5,2,3$, and 4.8 , measured in a reduced pressure environment, where the resulting change in density leads to a variation in the Rayleigh number of up to 5 decades from atmospheric conditions. These data will be used to identify trends in the physical behaviour of the system, as well as for validation of the completed modeling algorithms.

The parameter of interest in this research study is the total heat transfer rate, $Q$, through the enclosure from the inner to outer boundaries, determined from the temperature gradient in the fluid layer adjacent to the inner body surface:

$$
Q=\iint_{A_{i}}-\left.k \frac{\partial T}{\partial r}\right|_{r=r_{i}} d A_{i}
$$

The total heat transfer rate is non-dimensionalized by the Nusselt number defined using the overall temperature difference $\left(T_{i}-T_{o}\right)$ and a arbitrary scale length, $\mathcal{L}$ :

$$
N u_{\mathcal{L}}=\frac{Q \mathcal{L}}{k A_{i}\left(T_{i}-T_{o}\right)}
$$

The Rayleigh number is defined using the same overall temperature difference and length scale:

$$
R a_{\mathcal{L}}=\frac{g \beta\left(T_{i}-T_{o}\right) \mathcal{L}^{3}}{\nu \alpha}
$$

where all fluid properties are evaluated at the bulk temperature, $T_{b}$.

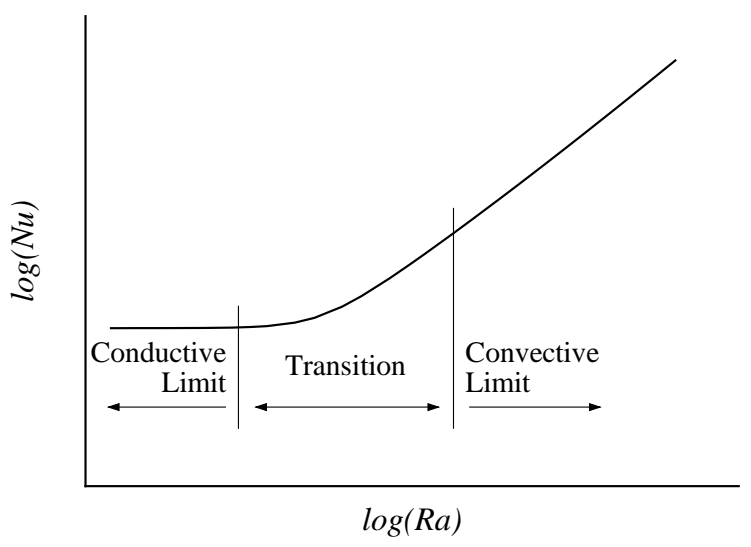

Fig. 2 Schematic of total heat transfer rate solution trends

The numerical data of Astill et al. ${ }^{6}$ for concentric spheres is used to describe the characteristics of the average heat transfer rate as a function of Rayleigh number. These authors present critical values for the Rayleigh number, $R a_{c r}$, where the dominant mode of heat transfer changes from conduction to convection. Three distinct regions are identified by Astill et al. ${ }^{6}$, as shown in Fig. 2.

- the conduction, or diffusive limit, $R a \ll R a_{c r}$, where heat transfer is dominated by conduction, independent of Rayleigh number and equivalent to the dimensionless conduction shape factor:

$$
N u_{\mathcal{L}}=S_{\mathcal{L}}^{\star}
$$

- the convective limit, $R a \gg R a_{c r}$, where heat transfer is dominated by convection at the inner and outer boundaries 
- the transition region where values of $N u$ move smoothly between the limiting cases, typically spanning one to two decades of $R a$ depending on geometry

The effective conductivity, $k_{e}$, is an alternate parameter used to quantify the average heat transfer rate in many studies. It is defined as the apparent value of thermal conductivity required for pure conduction through the enclosed region to be equal to convection. Previous researchers typically present results in terms of dimensionless effective conductivity, $k_{e} / k$, where $k$ is the actual thermal conductivity of the fluid. The dimensionless effective thermal conductivity is related to the Nusselt number and conduction shape factor $S_{L}^{\star}$ by:

$$
\frac{k_{e}}{k}=\frac{N u_{\mathcal{L}}}{S_{\mathcal{L}}^{\star}}, \frac{k_{e}}{k} \geq 1
$$

When dimensionless effective conductivity is used, all results approach a common asymptote, $k_{e} / k \rightarrow 1$, for the conductivity asymptote, $R a<R a_{c r}$.

Correlations of the experimental and numerical data presented in the literature by Bishop et al. ${ }^{2}$, Scanlan et al. ${ }^{4}$, Weber et al. ${ }^{5}$, Astill et al. ${ }^{6}$ and Wright and Douglass ${ }^{10}$ are valid over a limited range of Rayleigh number, typically corresponding to boundary layer convection. The only model in the literature for the concentric spheres that is valid for the full range of Rayleigh numbers is presented by Raithby and Hollands ${ }^{16}$ in terms of the dimensionless effective conductivity:

$$
\frac{k_{e}}{k}=\bar{C}_{c s} \frac{\delta^{1 / 4}}{d_{i} d_{o}} \frac{R a_{\delta}^{1 / 4}}{d_{i}^{-7 / 5}+d_{o}^{-7 / 5}}, \delta=\frac{d_{o}-d_{i}}{2}
$$

where the coefficient $\bar{C}_{c s}$ is a combination of the Prandtl number function and an empirically derived coefficient, C.

$$
\bar{C}_{c s}=C \cdot 0.56\left(\frac{P r}{0.846+P r}\right)^{1 / 4}
$$

Based on the measurements of Bishop et al. ${ }^{2}$ and Scanlan et al. ${ }^{4}$ for $d_{o} / d_{i}=2$, Raithby and Hollands ${ }^{16}$ select a value for the coefficient $C=1.32$ that provides good agreement between the model and the data. The model is recommended for use when $k_{e} / k>1$; for all other cases where values of $k_{e} / k$ are calculated that are less than one, the conduction limit, $k_{e} / k=1$ is used.

Comparison of the Raithby and Hollands ${ }^{16}$ model for the $d_{o} / d_{i}=2$ concentric spheres with the available numerical data for the transition region reveals that the model underpredicts the data by approximately $10 \%$ over the full range to the conduction limit. In the case of larger diameter ratios, such as the $d_{o} / d_{i}=4.8$ experimental data of Teertstra et al. ${ }^{15}$, the underprediction of the Raithby and Hollands ${ }^{16}$ model reached $25 \%$ near the transition region.

The cause of these differences between the data and the Raithby and Hollands ${ }^{16}$ model can be traced back to the original two term analysis of the heat transfer in the enclosure; one term that describes boundary layer convection at the limit of large Rayleigh number and the other term corresponding to the conduction shape factor solution. Using a piecewise function to combine these expressions results in a model that "switches" from convection to conduction at $R a_{c r}$, thereby neglecting the enhancement caused by combined conduction and convection in the transition region.

The goal of the current study is the development of an analytically based modeling procedure for natural convection in the concentric spherical enclosure that is valid over the full range of Rayleigh number, from the conduction limit to the laminar boundary layer convection limit. The model will be applicable for a wide range of aspect ratios, including the limiting case of the external convection solution, $d_{o} / d_{i} \rightarrow \infty$, and will not rely on empirically derived correlation coefficients.

\section{Model Development}

The previous models of Raithby and Hollands ${ }^{16}$ and Kuehn and Goldstein ${ }^{17}$ for the 2D circular annulus are based on a combination of two terms: one to quantify heat transfer due to convection and a second for the conduction limit. These terms can be combined using either the Churchill and Usagi ${ }^{18}$ composite technique, such as Kuehn and Goldstein ${ }^{17}$, or in a piecewise fashion, as in Raithby and Hollands ${ }^{16}$. This two term approach assumes that heat transfer in the enclosure occurs by one of two mechanisms; conduction through the gap or convection through boundary layers on the inner and outer surfaces. When laminar boundary layer convection is the dominant heat transfer mode, it is assumed that no interaction occurs between the inner and outer boundary layers. As the Rayleigh number decreases and the boundary layers approach each other, the two term model assumes a direct transition to conduction-dominated heat transfer occurs.

The experimental data of Teertstra et al. ${ }^{15}$ is used to demonstrate that a two term model is inadequate to describe the heat transfer process in the enclosure. For natural convection from a heated body in a full-space domain, Yovanovich ${ }^{19}$ recommends linear superposition of the convective asymptote with the diffusive limit. Assuming this relationship holds for the enclosure, it is possible to predict the portion of the heat transfer due to convection from experimental data, $N u_{\sqrt{A_{i}}}$, using the following relationship:

$$
N u_{\text {conv }}=N u_{\sqrt{A_{i}}}-S_{\sqrt{A_{i}}}^{\star}
$$




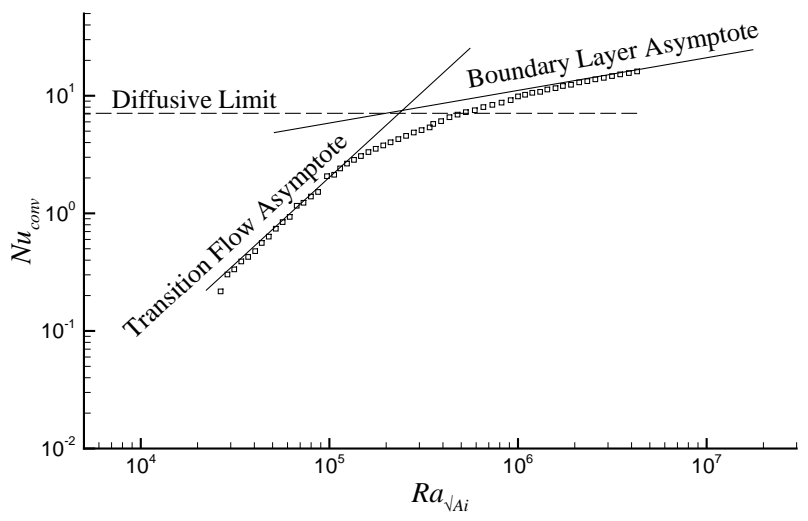

Fig. 3 Convection-only reduced experimental data ${ }^{15}$ : concentric spheres, $d_{o} / d_{i}=2$

where the conduction shape factor $S_{\sqrt{A_{i}}}^{\star}$ is available from the exact solution for conduction in the spherical shell. Convection-only data, $N u_{\text {conv }}$ computed by Eq. (7) are presented in Fig. 3 for $d_{o} / d_{i}=2$.

Figure 3 clearly demonstrates that, once the conduction portion of the heat transfer has been reduced from the data, the relationship between $N u_{\text {conv }}$ and $R a_{\sqrt{A_{i}}}$ approaches a second asymptotic solution when $R a_{\sqrt{A_{i}}}<$ $3 \times 10^{5}$. This indicates that an additional term should be incorporated in the existing models to account for changes that occur in the transition region, where boundary layers merge, a velocity distribution is established in the core region, and a transition flow pattern emerges. The general form of this three term model is:

$$
N u_{\sqrt{A_{i}}}=S_{\sqrt{A_{i}}}^{\star}+\left(N u_{\mathrm{tr}}^{-n}+N u_{\mathrm{bl}}^{-n}\right)^{-1 / n}
$$

where the asymptotic solutions for transition flow, $N u_{\mathrm{tr}}$, and boundary layer flow, $N u_{\mathrm{bl}}$, are combined using the Churchill and Usagi ${ }^{18}$ composite technique. The combination parameter, $n$, will be selected based on a comparison with the available experimental data.

The square root of the inner body surface area, $\sqrt{A_{i}}$, has been selected as the characteristic length for the dimensionless quantities based on two criteria. First, for large aspect ratio enclosures, $d_{o} / d_{i} \rightarrow \infty$, heat transfer is controlled by convection at the inner boundary; therefore the scale length should be related to inner dimensions only and be independent of the outer boundary geometry. Second, Yovanovich ${ }^{19}$ and Jafarpur ${ }^{20}$ have shown that, when $\sqrt{A_{i}}$ is used, complex geometries can be successfully modeled using equivalent bodies of similar aspect ratio that are more easily characterized.

In the following sections component models for each of the asymptotes in Eq. (8) will be developed.

Conduction Shape Factor The thermal resistance for the concentric spherical shell is determined from a sim- ple conduction analysis in spherical coordinates (Incropera and DeWitt ${ }^{21}$ ):

$$
R=\frac{1}{2 \pi k}\left(\frac{1}{d_{i}}-\frac{1}{d_{o}}\right)
$$

Incorporating the gap thickness $\delta$ by substituting $d_{o}=$ $d_{i}+2 \delta$ gives:

$$
R=\frac{\delta}{\pi k d_{i}\left(d_{i}+2 \delta\right)}
$$

The equivalent dimensionless conduction shape factor is determined by:

$$
S_{\sqrt{A_{i}}}^{\star}=\frac{1}{k \sqrt{A_{i}} R}=\frac{\sqrt{\pi} d_{i}}{\delta}+2 \sqrt{\pi}
$$

Equation (11) represents a linear combination of two limiting cases: for small gap spacing, $\delta \ll d_{i}$, the first term is dominant, corresponding to $1 \mathrm{D}$, planar resistance; as the gap spacing becomes large, $S_{\sqrt{A_{i}}}^{\star}$ tends to the constant value of a sphere in a full space region, $2 \sqrt{\pi}$.

Laminar Boundary Layer Convection At the limit of large Rayleigh number, assuming the fluid in the core region is of uniform temperature and that the gap spacing $\delta$ is large compared to the boundary layer thickness, the convective heat transfer through the enclosure can be modeled as a series combination of two thermal resistances:

$$
R_{\mathrm{conv}}=R_{i}+R_{o}
$$

where $R_{i}$ and $R_{O}$ refer to thermal resistance due to convection at the inner and outer boundaries, respectively. This total resistance is expressed as a Nusselt number:

$$
N u_{\mathrm{bl}}=\frac{1}{k \sqrt{A_{i}}} \frac{1}{\left(R_{i}+R_{o}\right)}=\frac{1}{k \sqrt{A_{i}}} \frac{1}{R_{i}} \frac{1}{\left(1+R_{o} / R_{i}\right)}
$$

From the definitions of the thermal resistances, the ratio $R_{o} / R_{i}$ in Eq. (13) is recast in terms of dimensionless bulk temperature $\phi$ :

$$
R_{i}=\frac{T_{i}-T_{b}}{Q}, R_{o}=\frac{T_{b}-T_{o}}{Q}, \frac{R_{i}}{R_{o}}=\frac{T_{i}-T_{b}}{T_{b}-T_{o}}=\phi
$$

Substituting $\phi$ and $N u_{i}=1 /\left(k \sqrt{A_{i}} R_{i}\right)$ into Eq. (13) gives:

$$
N u_{b l}=\frac{N u_{i}}{1+1 / \phi}
$$

The convective component at the inner body, $N u_{i}$ is modeled using the method presented by Yovanovich ${ }^{19}$ and Jafarpur $^{20}$ for natural convection from isothermal, arbitrarily shaped bodies:

$$
N u_{\sqrt{A}}=F(P r) G_{\sqrt{A}} R a_{\sqrt{A}}^{1 / 4}
$$


where the Prandtl number function, $F(P r)$, valid for all isothermal body shapes is presented by Churchill and Churchill $^{22}$ :

$$
F(P r)=\frac{0.67}{\left[1+(0.5 / P r)^{9 / 16}\right]^{4 / 9}}
$$

and the body gravity function, $G_{\sqrt{A}}$ is presented by Lee et al. ${ }^{23}$ :

$$
G_{\sqrt{A}}=\left[\frac{1}{A} \iint_{A}\left(\frac{P \sin \theta}{\sqrt{A}}\right)^{1 / 3} d A\right]^{3 / 4}
$$

For the inner body, the convection model is

$$
\begin{aligned}
N u_{i} & =F(P r) G_{\sqrt{A}} R a_{i}^{1 / 4} \\
R a_{i} & =\frac{g \beta\left(T_{i}-T_{o}\right)\left(\sqrt{A_{i}}\right)^{3}}{v \alpha} \\
& =R a_{\sqrt{A_{i}}}\left(\frac{T_{i}-T_{b}}{T_{b}-T_{o}}\right)^{1 / 4}
\end{aligned}
$$

Substituting Eqs. (19) and (20) into Eq. (15) and simplifying yields:

$$
N u_{b l}=\frac{F(P r) G_{\sqrt{A}} R a_{\sqrt{A_{i}}}^{1 / 4}}{(1+1 / \phi)^{5 / 4}}
$$

Evaluating the dimensionless bulk temperature using the natural convection modeling procedure from Eq. (16) provides the following relationship between $\phi$ and the enclosure geometry:

$$
\begin{aligned}
\phi & =\frac{R_{i}}{R_{o}}=\frac{\sqrt{A_{o}} N u_{o}}{\sqrt{A_{i}} N u_{i}} \\
& =\frac{\sqrt{A_{o}}}{\sqrt{A_{i}}} \frac{G_{\sqrt{A_{o}}}}{G_{\sqrt{A_{i}}}} \frac{\left(T_{b}-T_{o}\right)^{1 / 4}}{\left(T_{i}-T_{b}\right)^{1 / 4}} \frac{\left(\sqrt{A_{o}}\right)^{3 / 4}}{\left(\sqrt{A_{i}}\right)^{3 / 4}} \\
& =\left(\frac{A_{o}}{A_{i}}\right)^{7 / 4} \frac{G_{\sqrt{A_{o}}}}{G_{\sqrt{A_{i}}}} \frac{1}{\phi^{1 / 4}}
\end{aligned}
$$

Solving Eq. (22) for $\phi$ and substituting into Eq. (21) results in the general model for the boundary layer flow convective asymptote:

$$
N u_{\mathrm{bl}}=\frac{F(\operatorname{Pr}) G_{\sqrt{A_{i}}} \operatorname{Ra}_{\sqrt{A_{i}}}^{1 / 4}}{\left[1+\left(\frac{A_{i}}{A_{o}}\right)^{7 / 10}\left(\frac{G_{\sqrt{A_{i}}}}{G_{\sqrt{A_{o}}}}\right)^{4 / 5}\right]^{5 / 4}}
$$

For the particular problem of the concentric spherical enclosure, where $A_{i}=\pi d_{i}^{2}$ and $A_{o}=\pi d_{o}^{2}$, and the body gravity functions evaluate to:

$$
G_{\sqrt{A_{o}}}=G_{\sqrt{A_{i}}}=1.014
$$

The general expression for the laminar boundary layer asymptote can be simplified as follows:

$$
N u_{\mathrm{bl}}=\frac{F(\operatorname{Pr})(1.014) R a_{\sqrt{A_{i}}}^{1 / 4}}{\left[1+\left(d_{i} / d_{o}\right)^{7 / 5}\right]^{5 / 4}}
$$

All thermofluid properties are evaluated at the bulk fluid temperature, determined by re-arranging Eq. (22) in terms of $T_{b}$ :

$$
T_{b}=\frac{T_{i}+T_{o}\left(d_{o} / d_{i}\right)^{7 / 5}}{1+\left(d_{o} / d_{i}\right)^{7 / 5}}
$$

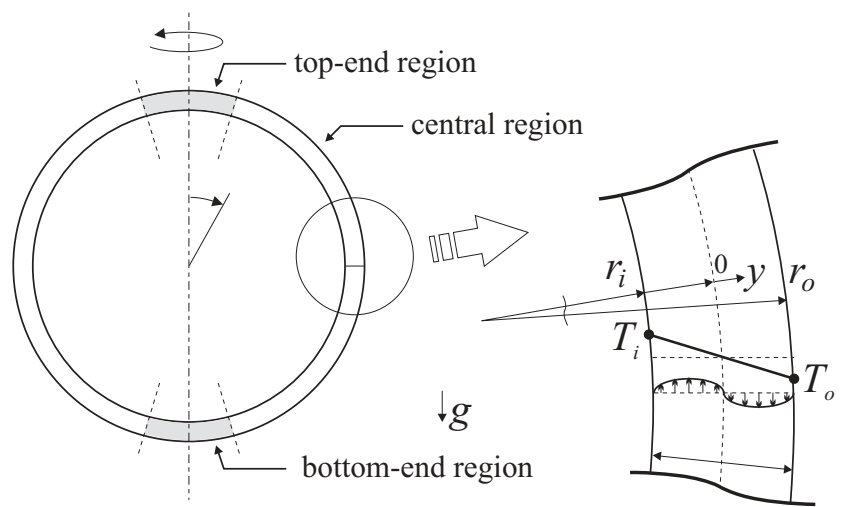

Fig. 4 Transition flow in concentric spherical enclosure

Transition Flow Convection The third and final asymptote corresponds to convective heat transfer that occurs in the transition between the conduction and laminar boundary layer convection limits. As the Rayleigh number decreases, the boundary layers on the inner and outer surfaces of the enclosure grow and eventually merge along the midplane when $R a<R a_{c r}$. For Rayleigh number at or below this critical value, the enclosed region can be divided into three regions as shown in Fig. 4; the top-end and bottom-end regions (adjacent to the axis of symmetry) and the central region. The heat transfer in the central region is dominated by conduction in the radial direction, and the energy equation reduces to:

$$
\frac{1}{r^{2}} \frac{d}{d r}\left(r^{2} \frac{d T}{d r}\right)=0
$$

This radial temperature distribution results in a small, buoyancy-induced flow within the region; an upwards flow with respect to the gravity vector in the inner half of the shell and a downwards flow in the outer half. Assuming steady state, constant property axisymmetric flow with no radial velocity component, the momentum equation simplifies to:

$$
\frac{1}{r^{2}} \frac{d}{d r}\left(r^{2} \frac{d u}{d r}\right)=-\frac{g \beta}{v} \sin \theta\left(T-T_{b}\right)
$$




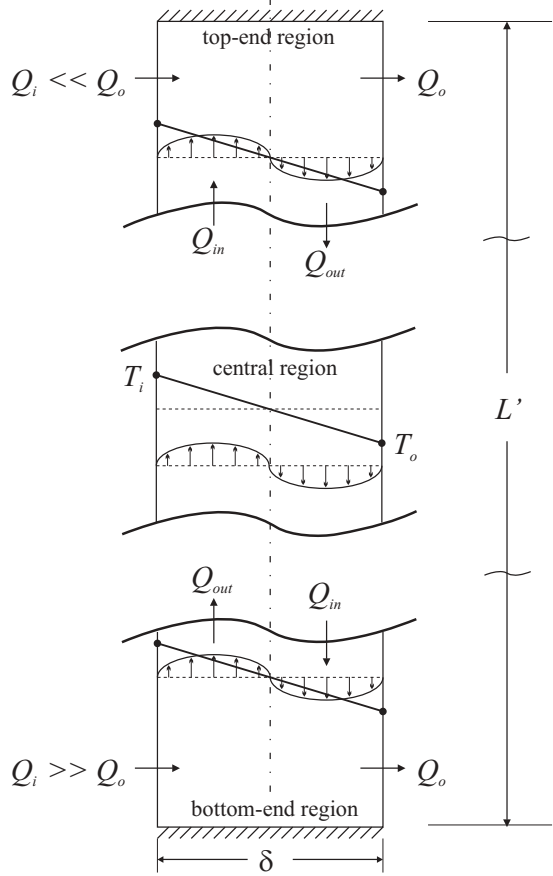

Fig. 5 Schematic of vertical cavity model regions

where $T_{b}$ is the midplane temperature. For the limiting case of narrow gap spacing with respect to the inner and outer boundary dimensions, $\delta \ll d_{i}, d_{o}$, the governing equations in spherical coordinates can be related to the equivalent problem of flow between vertical plates by defining a local Cartesian coordinate system, as shown in Fig. 4. The radial coordinate is related to the local Cartesian coordinate by:

$$
r=r_{i}+\frac{\delta}{2}+y, \quad-\frac{\delta}{2} \leq y \leq \frac{\delta}{2}
$$

and the temperature gradient in spherical coordinates is related to the local coordinate system by:

$$
\frac{d T}{d r}=\frac{d T}{d y} \frac{d y}{d r}=\frac{d T}{d y} \frac{d}{d r}\left(r-r_{i}-\frac{\delta}{2}\right)=\frac{d T}{d y}
$$

Therefore, at the limit $\delta \ll r_{i}$ the energy and momentum equations are transformed to the local coordinate system:

$$
\begin{gathered}
\frac{1}{r^{2}} \frac{d}{d r}\left(r^{2} \frac{d T}{d r}\right) \rightarrow \frac{d^{2} T}{d y^{2}}=0 \\
\frac{1}{r^{2}} \frac{d}{d r}\left(r^{2} \frac{d u}{d r}\right) \rightarrow \frac{d^{2} u}{d y^{2}}=-\frac{g_{e} \beta}{v} \sin \theta\left(T-T_{b}\right)
\end{gathered}
$$

where $g_{e}=g \sin \theta$ is the effective gravitation coefficient.

Bird et al. ${ }^{24}$ and Rohsenow and Choi ${ }^{25}$ present a solution of these governing equations for the vertical, differentially heated channel. Solving the energy equation, Eq. (31) for isothermal boundary conditions $T(-\delta / 2)=$ $T_{i}$ and $T(\delta / 2)=T_{o}$ gives:

$$
T-T_{b}=-\frac{y}{\delta / 2}\left(T_{i}-T_{b}\right), T_{b}=\frac{T_{i}+T_{o}}{2}
$$

Substituting this expression into the momentum equation, Eq. (32), and solving with a no-slip condition at the wall yields:

$$
u=\frac{g_{e} \beta}{12 v}\left(T_{i}-T_{o}\right)\left(\frac{\delta}{2}\right)^{2}\left[\left(\frac{y}{\delta / 2}\right)^{3}-\frac{y}{\delta / 2}\right]
$$

In the central region, the heat transfer is due to conduction only, and the linear temperature distribution results in fluid motion due to buoyancy effects. The addition of the top-end and bottom-end regions to the analysis, as shown in Fig. 5, transforms the vertical parallel plate problem to that of natural convection in a vertical cavity. Batchelor ${ }^{26}$ and Eckert and Carlson ${ }^{27}$ present a method for analysis of convective heat transfer in the vertical cavity based on an enthalpy balance within the control volumes formed at the top-end and bottom-end regions, as shown in Fig. 5. For the bottom-end control volume, an enthalpy balance is performed that equates the heat transfer by conduction, $Q_{i}$ and $Q_{o}$, with the heat transfer through advection by the fluid entering and exiting the control volume from the inner and outer halves of the central region, $Q_{\text {in }}$ and $Q_{\text {out }}$. The enthalpy flux from the central region, $Q_{\text {out }}$, is determined based on an integration of the temperature and velocity distributions. For the inner half of the cavity:

$$
Q_{\text {out }}=\int_{-\delta / 2}^{0} \rho c_{p} W^{\prime} u\left(T-T_{o}\right) d y
$$

where $W^{\prime}$ is the width of the control volume and the temperature rise is defined with respect to the outer wall temperature, $T_{o}$. Substituting Eqs. (33) and (34) for the temperature and velocity distributions and solving the integral yields:

$$
Q_{\text {out }}=\frac{23}{120} \frac{\rho c_{p} W^{\prime} g_{e} \beta\left(T_{i}-T_{o}\right)^{2} \delta^{3}}{96 v}
$$

Repeating the analysis for the enthalpy flux for the outer half of the control volume gives:

$$
Q_{i n}=\frac{7}{120} \frac{\rho c_{p} W^{\prime} g_{e} \beta\left(T_{i}-T_{o}\right)^{2} \delta^{3}}{96 v}
$$

For the bottom-end control volume it is assumed that the boundary layer on the inner, heated surface is much thinner than on the outer, cooled surface, such that $Q_{i} \gg Q_{o}$. Therefore, the total enthalpy balance for the bottom-end region is:

$$
Q_{i}=Q_{\text {out }}-Q_{\text {in }}=\frac{\rho c_{p} W^{\prime} g_{e} \beta\left(T_{i}-T_{o}\right)^{2} \delta^{3}}{720 v}
$$


The analysis for the top-end region control volume yields an equivalent result, with the total heat transfer to the cooled outer surface given by:

$$
Q_{o}=Q_{\text {in }}-Q_{\text {out }}=\frac{\rho c_{p} W^{\prime} g_{e} \beta\left(T_{i}-T_{o}\right)^{2} \delta^{3}}{720 v}
$$

Therefore, the total heat transfer rate due to convection for the transition flow asymptote is equivalent to $Q_{i}$ in the bottom-end region and $Q_{o}$ in the top-end region.

The total heat transfer rate is non-dimensionalized by the Nusselt number:

$$
N u_{\mathrm{tr}}=\frac{Q \sqrt{A_{i}}}{k L^{\prime} W^{\prime}\left(T_{i}-T_{o}\right)}
$$

where $L^{\prime} \times W^{\prime}$ is the cross sectional area of the equivalent cavity. Substituting $Q$ from the enthalpy balance, Eq. (38), and simplifying the resulting expression yields:

$$
\begin{aligned}
N u_{\mathrm{tr}} & =\frac{1}{720} \frac{\sqrt{A_{i}}}{L^{\prime}} \frac{g_{e} \beta\left(T_{i}-T_{o}\right) \delta^{3}}{\nu \alpha} \\
& =\frac{1}{720} \frac{\sqrt{A_{i}}}{L^{\prime}} \frac{g_{e}}{g}\left(\frac{\delta}{\sqrt{A_{i}}}\right)^{3} R a_{\sqrt{A_{i}}}
\end{aligned}
$$

The effective gravitation coefficient is calculated based an area-weighted integration over a spherical surface at the midplane of the enclosed space:

$$
g_{e}=\frac{1}{A} \iint_{A}(g \sin \theta) r^{2} \sin \theta d A=\frac{\pi}{4}
$$

The ratio of the gap spacing to the inner surface area is easily calculated for the concentric spheres:

$$
\frac{\delta}{\sqrt{A_{i}}}=\frac{d_{o}-d_{i}}{2 \sqrt{\pi} d_{i}}
$$

The length of the equivalent cavity is determined from an arithmetic average of the inner and outer dimensions, $L^{\prime}=\left(L_{o}+L_{i}\right) / 2$, where effective lengths $L_{o}$ and $L_{i}$ are required that reflect the dimensions and shape of the boundaries. Defining the width $W$ according to the maximum perimeter of the body on a plane perpendicular to $g$ provides an upper bound for the Nusselt number:

$$
W_{U B}=\pi d, L_{U B}=\frac{A}{W_{U B}}=d
$$

The lower bound results from defining the effective length as the distance from the bottom to top stagnation points, or half the perimeter of the body on a plane parallel to the gravity vector:

$$
L_{L B}=\pi d / 2
$$

The bounds on effective length are combined using a geometric mean:

$$
\begin{aligned}
L^{\prime} & =\sqrt{\left(L_{o}+L_{i}\right)_{U B}\left(L_{o}+L_{i}\right)_{L B}} \\
& =\sqrt{\frac{1}{2} \cdot \frac{\pi}{4}}\left(d_{o}+d_{i}\right)
\end{aligned}
$$

Substituting the relationships for effective gravitation coefficient and effective length into Eq. (41) completes of the model for the transition flow asymptote:

$$
N u_{\mathrm{tr}}=\frac{\sqrt{2 / \pi}}{11520} \frac{\left(d_{o} / d_{i}-1\right)^{3}}{\left(d_{o} / d_{i}+1\right)} R a_{\sqrt{A_{i}}}
$$

Model Summary The three term natural convection model for the concentric spherical enclosure is summarized as follows:

$$
\begin{aligned}
N u_{\sqrt{A_{i}}}= & \left(\frac{\sqrt{\pi} d_{i}}{\delta}+2 \sqrt{\pi}\right) \\
+ & {\left[\left(\frac{\sqrt{2 / \pi}\left(d_{o} / d_{i}-1\right)^{3}}{11520} \frac{\left.d_{o} / d_{i}+1\right)}{A_{A_{i}}}\right)^{-2}\right.} \\
& \left.+\left(\frac{F(\operatorname{Pr})(1.014) \operatorname{Ra} \sqrt{A_{i}}}{\left[1+\left(d_{i} / d_{o}\right)^{7 / 5}\right]^{5 / 4}}\right)^{-2}\right]^{-1 / 2} \\
N u_{\sqrt{A_{i}}} & \equiv \frac{Q}{k \sqrt{A_{i}}\left(T_{i}-T_{o}\right)} \\
F(\operatorname{Pr}) & =0.67\left[1+(0.5 / \operatorname{Pr})^{9 / 16}\right]^{-4 / 9} \\
R a_{\sqrt{A_{i}}} & \equiv \frac{g \beta\left(T_{i}-T_{o}\right)\left(\sqrt{A_{i}}\right)^{3}}{v \alpha}
\end{aligned}
$$

where a combination parameter value $n=2$ was selected that provides a good fit to the experimental data of Teertstra et al. ${ }^{15}$

\section{Model Validation}

The natural convection model developed in the previous section and summarized in Eqs. (48) - (51) is validated using the experimental data of Teertstra et al. ${ }^{15}$ and other numerical and experimental data from the literature for a wide range of diameter ratios and Rayleigh number.

Figure 6 compares the convection-only portion of the model with the experimental data of Teertstra et al. ${ }^{15}$ reduced using Eq. (7), where $S_{\sqrt{A_{i}}}^{\star}$ is the exact solution for conduction shape factor in the concentric spherical shell. This comparison with the composite model for the convection terms is used in the selection of a combination parameter, $n=2$, the provides good agreement of the model with the data throughout the transition region. 


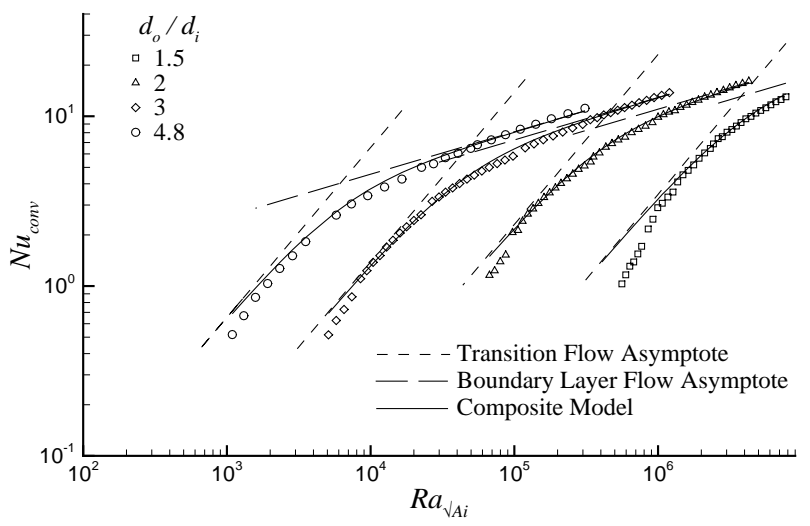

Fig. 6 Convection-only model validation with experimental data of Teertstra et al. ${ }^{15}$

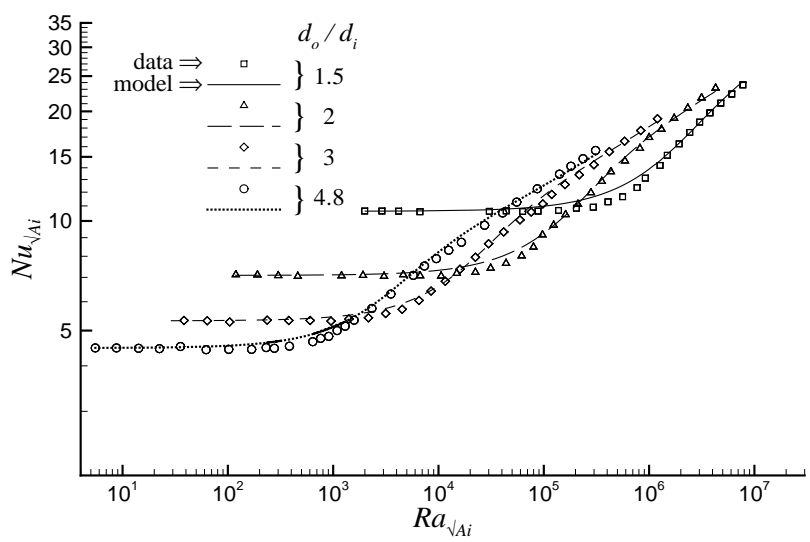

Fig. 7 Model validation with Teertstra et al. ${ }^{15}$

Table 1 Comparison of model predictions versus data

(Teertstra et al. ${ }^{15}$ )

\begin{tabular}{|c|c|c|}
\hline$d_{o} / d_{i}$ & $R M S \%$ & max \% \\
\hline 1.5 & 4.3 & 7.3 \\
2 & 3.0 & 5.8 \\
3 & 2.3 & 4.9 \\
4.8 & 2.8 & 4.9 \\
\hline
\end{tabular}

The larger differences shown in Fig. 6 as the Rayleigh number decreases further are due to uncertainty in the experimental data, which becomes more significant as $N u_{\sqrt{A_{i}}} \rightarrow S_{\sqrt{A_{i}}}^{\star}$ and $N u_{\text {conv }} \rightarrow 0$.

The full model, Eqs. (48) - (51), is compared with the experimental data of Teertstra et al. ${ }^{15}$ for four different concentric spherical enclosures, $d_{o} / d_{i}=1.5,2,3$, and 4.8 in Fig. 7. This plot shows the excellent agreement between the model and the data, with the model successfully following the trends of the data and the transition from conduction to convection-dominated heat
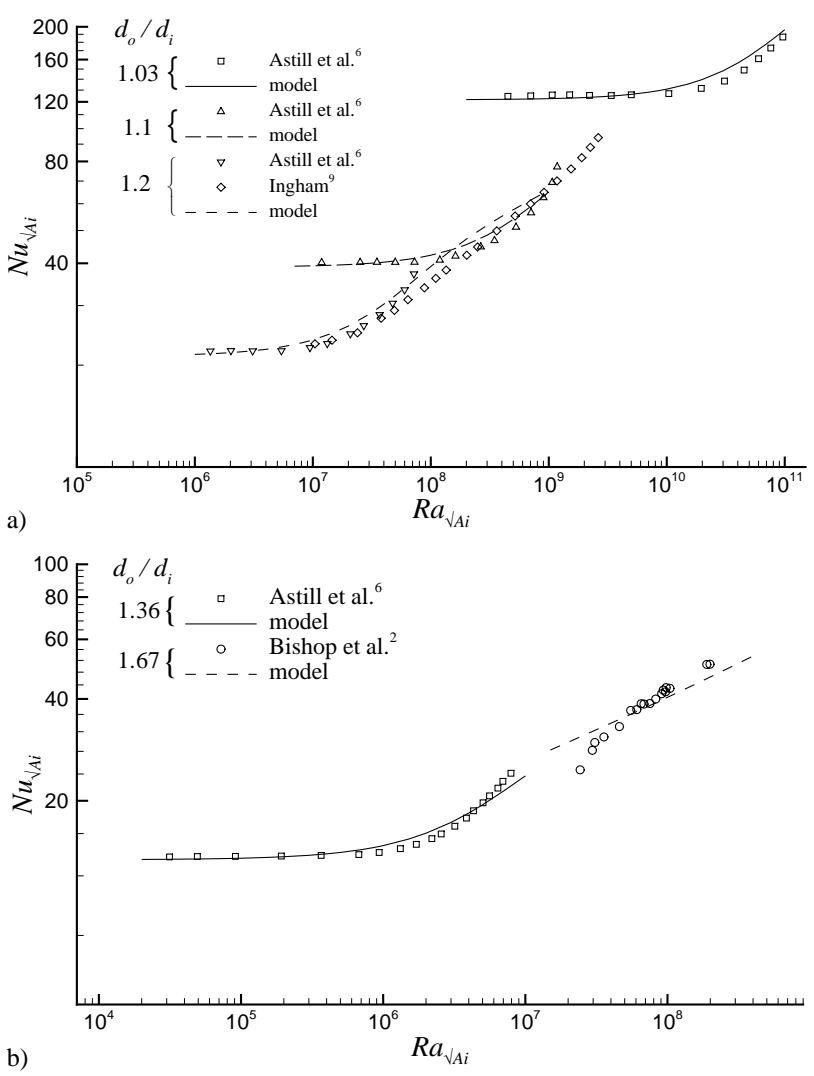

b)

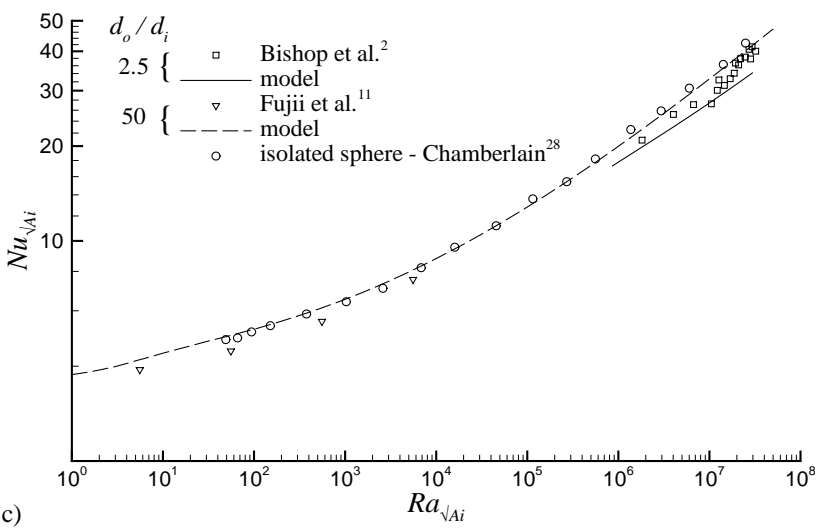

Fig. 8 Model validation with previous data:

a) $d_{o} / d_{i} \leq 1.2$; b) $1.25 \leq d_{o} / d_{i}<2$; c) $d_{o} / d_{i} \geq 2.5$

transfer occurring at different Rayleigh numbers depending on the diameter ratio. The overall RMS difference between the data and the model is $3-4 \%$, with a maximum difference of $7 \%$. A full list of the percent differences is given in Table 1.

Figure 8 compares the model with existing numerical and experimental data from the literature for a wide range of diameter ratio; from the $d_{o} / d_{i}=1.03$ numerical data of Astill et al. ${ }^{6}$ presented in Fig. 8 a) to the $d_{o} / d_{i}=50$ numerical data of Fujii et al. ${ }^{11}$ in Fig. 8 c). The model and data are in good agreement in all cases presented in 


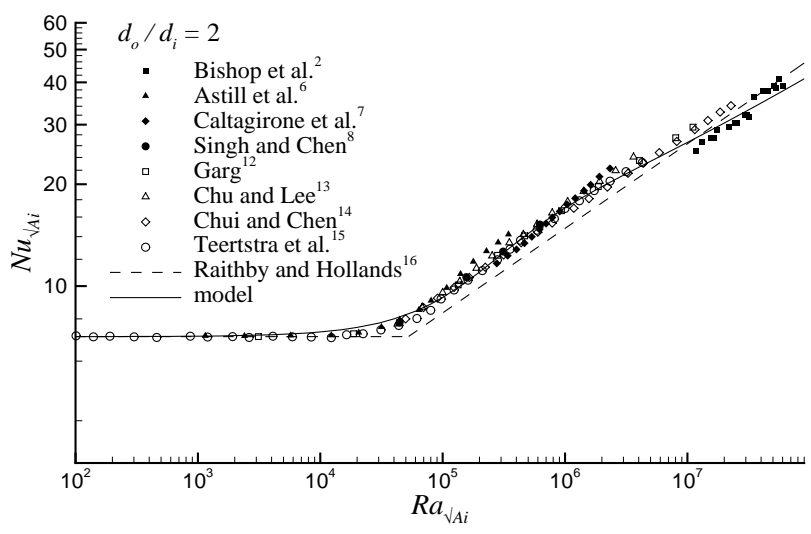

Fig. 9 Model validation with previous data and models:

$$
d_{o} / d_{i}=2
$$

Fig. 8 a) - c). The large amount of scatter in the experimental data of Bishop et al. ${ }^{2}$ makes is difficult to provide an accurate fit of all the data points; however both Figs. 8 b) and c) demonstrate that the model is in good agreement with the majority of the data. Figure $8 \mathrm{c}$ ) also compares the model with the experimental measurements of Chamberlain ${ }^{28}$ for an isothermal sphere in a full space domain, a limiting case of the concentric spherical enclosure, $d_{o} / d_{i} \rightarrow \infty$, for which the three term model is in excellent agreement.

The majority of the numerical and experimental data presented in the literature involves the $d_{o} / d_{i}=2$ concentric spherical enclosure, and all available data for this configuration are compared with the model predictions in Fig. 9. All of these data represent the result of numerical simulations with the exception of the experimental data of Bishop et al. ${ }^{2}$. All of these data are in good agreement with each other, with the majority of the data within $\pm 5 \%$ for $R a_{\sqrt{A_{i}}}<10^{6}$. As can be seen from Fig. 9 the three term natural convection model provides excellent agreement with the data over the full range of Rayleigh number, from the numerical conduction limit data of $\mathrm{Garg}^{12}$ through the transition region to the experimental laminar boundary layer data of Bishop et al. ${ }^{2}$.

Also included in Fig. 9 is the two term model of Raithby and Hollands ${ }^{16}$, which provides an effective fit of the data for high values of Rayleigh numbers, $R a_{\sqrt{A_{i}}} \approx$ $10^{7}$; however, for $R a_{\sqrt{A_{i}}} \leq 10^{6}$ this model underpredicts the data by approximately $10 \%$ over the full range to the conduction limit. The piecewise method used by Raithby and Hollands ${ }^{16}$ results in a model that neglects the contribution of conduction to the heat transfer for intermediate values of Rayleigh number in the transition region.

The model and data of Teertstra et al. ${ }^{15}$ for $d_{o} / d_{i}=$ 4.8 are compared with the model of Raithby and Hollands ${ }^{16}$ in Fig. 10. As in the previous plot, Fig. 10 demonstrates that the two term model of Raithby and

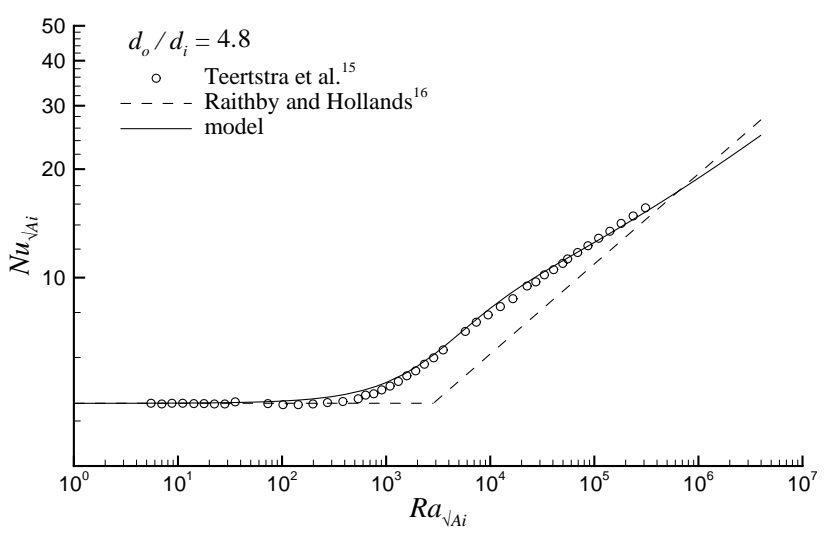

Fig. 10 Model validation with previous data and models:

$$
d_{o} / d_{i}=4.8
$$

Hollands ${ }^{16}$ underpredicts both the model and the data, with a maximum difference of $25 \%$ at $R a_{c r}$.

\section{Summary}

A model has been developed for natural convection heat transfer in the concentric spherical enclosure with isothermal conditions on the inner (heated) and outer (cooled) boundaries. The model is based on three limiting case solutions, corresponding to the diffusive limit, laminar boundary layer convection and transition flow convection. A full validation with published numerical and experimental results has demonstrated the effectiveness of the model for a wide range of diameter ratios and Rayleigh number, with an average RMS difference of $2-3 \%$. The modeling procedure developed for this fundamental geometry will provide the basis for future analyses of more complex enclosure configurations.

\section{Acknowledgments}

The authors acknowledge the continued financial support of Materials and Manufacturing Ontario (MMO) and the Centre for Microelectronics Assembly and Packaging (CMAP).

\section{References}

${ }^{1}$ Bishop, E.H., Kolflat, R.S., Mack, L.R. and Scanlan, J.A., "Convective Heat Transfer Between Concentric Spheres," Proceedings of the 1964 Heat Transfer and Fluid Mechanics Institute, Stanford University Press, Stanford, CA, pp. 69 - 80, 1964.

2 Bishop, E.H., Mack, L.R. and Scanlan, J.A., "Heat Transfer by Natural Convection Between Concentric 
Spheres," International Journal of Heat and Mass Transfer, Vol. 9, pp. 649 - 662, 1966.

${ }^{3}$ Mack, L.R. and Hardee, H.C., "Natural Convection between Concentric Spheres at Low Rayleigh Numbers," International Journal of Heat and Mass Transfer, Vol. 11, pp. 387 - 396, 1968.

${ }^{4}$ Scanlan, J. A., Bishop, E. H. and Powe, R. E., "Natural Convection Heat Transfer Between Concentric Spheres," International Journal of Heat and Mass Transfer, Vol. 13, pp. 1857 - 1872, 1970.

5 Weber, N., Powe, R. E., Bishop, E. H. and Scanlan, J. A., "Heat Transfer by Natural Convection Between Vertically Eccentric Spheres," Transactions of the ASME: Journal of Heat Transfer, Vol. 95, pp. 47 - 52, 1973.

6 Astill, K.N., Leong, H. and Martorana, R., "A Numerical Solution for Natural Convection in Concentric Spherical Annuli," Natural Convection In Enclosures, ASME-HTD Vol. 8, pp. 105 - 113, 1980.

7 Caltagirone, J.-P., Combarnous, M. and Mojtabi, A., "Natural Convection between Two Concentric Spheres: Transition toward a Multicellular Flow," Numerical Heat Transfer, Vol. 3, pp. 107 - 114, 1980.

${ }^{8}$ Singh, S.H. and Chen, J., "Numerical Solution for Free Convection between Concentric Spheres at Moderate Grashof Numbers," Numerical Heat Transfer, Vol. 3, pp. 441 - 459, 1980.

9 Ingham, D.B., "Heat Transfer by Natural Convection between Spheres and Cylinders," Numerical Heat Transfer, Vol. 4, pp. 53 - 67, 1981.

10 Wright, J.L. and Douglass, R.W., "Natural Convection in Narrow-gap, Spherical Annuli," International Journal of Heat and Mass Transfer, Vol. 29, No. 5, pp. 725-739, 1986.

11 Fujii, M., Takamatsu, H. and Fujii, T., "A Numerical Analysis of Free Convection around an Isothermal Sphere (Effects of Space and Prandtl Number)," Proceedings of the 1987 ASME/JSME Thermal Engineering Joint Conference, Vol. 4, pp. 55 - 60, 1987.

12 Garg, V.K., "Natural Convection between Concentric Spheres," International Journal of Heat and Mass Transfer, Vol. 35, pp. 1935 - 1945, 1992.

13 Chu, H-S. and Lee, T-S., "Transient Natural Convection Heat Transfer between Concentric Spheres," International Journal of Heat and Mass Transfer, Vol. 36, pp. 3159 - 3170, 1993.

${ }^{14}$ Chiu, C.P. and Chen, W.R., "Transient Natural Convection Heat Transfer between Concentric and Vertically Eccentric Spheres," International Journal of Heat and Mass Transfer, Vol. 39, pp. 1439 - 1452, 1996.

15 Teertstra, P., Yovanovich, M.M. and Culham, J.R., "Natural Convection Measurements for a Concentric Spherical Enclosure," 2002 IMECE Conference, November 17, New Orleans, LA.

16 Raithby, G.D. and Hollands, K.G.T., "A General
Method of Obtaining Approximate Solutions to Laminar and Turbulent Free Convection Problems," Advances in Heat Transfer, Editors T.F. Irvine Jr. and J.P. Hartnett, Academic Press, New York, Vol. 11, pp. 265 - 315, 1975.

17 Kuehn, T.H. and Goldstein, R.J., "Correlating Equations for Natural Convection Heat Transfer Between Horizontal Circular Cylinders," International Journal of Heat and Mass Transfer, Vol. 19, pp. 1127 - 1134, 1976b

${ }^{18}$ Churchill, S.W. and Usagi, R., "A General Expression for the Correlation of Rates of Transfer and Other Phenomenon," American Institute of Chemical Engineers Journal, Vol. 18, pp. 1121 - 1128, 1972.

19 Yovanovich, M.M., "New Nusselt and Sherwood Numbers for Arbitrary Isopotential Geometries at Near Zero Peclet and Rayleigh Numbers," AIAA 22nd Thermophysics Conference, AIAA-87-1643, 1987.

20 Jafarpur, K., “Analytical and Experimental Study of Laminar Free Convective Heat Transfer from Isothermal Convex Bodies of Arbitrary Shape," Ph.D. Thesis, Department of Mechanical Engineering, University of Waterloo, 1992.

21 Incropera, F. P. and DeWitt, D. P., Fundamentals of Heat and Mass Transfer, 4th ed., Wiley, New York, pp. 96 - 99, 114 - 118, 1996.

22 Churchill, S.W. and Churchill, R.U., "A Comprehensive Correlating Equation for Heat and Component Heat Transfer by Free Convection," American Institute of Chemical Engineers Journal, Vol. 21, pp. 604 - 606, 1975.

${ }^{23}$ Lee, S., Yovanovich, M. M. and Jafarpur, K., "Effects of Geometry and Orientation on Laminar Natural Convection Heat Transfer from Isothermal Bodies," Journal of Thermophysics and Heat Transfer, Vol. 5, pp. 208 216, 1991.

${ }^{24}$ Bird, R.B., Stewart, W.E. and Lightfoot, E.N., Transport Phenomena, John Wiley and Sons, New York, NY, pp. 297 - 300, 1960.

${ }^{25}$ Rohsenow, W.M. and Choi, H.Y., Heat, Mass and Momentum Transfer, Prentice-Hall, NJ, pp. 143 - 146, 1961. 26 Batchelor, G.K., "Heat Transfer by Free Convection Across a Closed Cavity Between Vertical Boundaries at Different Temperatures," Quarterly of Applied Mathematics, Vol. 12, No. 3, pp. 209 - 223, 1954.

27 Eckert, E.R.G. and Carlson, W.O., "Natural Convection in an Air Layer Enclosed Between Two Vertical Plates with Different Temperatures" International Journal of Heat and Mass Transfer, Vol. 2, pp. 106 - 120, 1961.

28 Chamberlain, M.J., "Free Convection Heat Transfer from a Sphere, Cube and Vertically Aligned Bi-Sphere," M.A.Sc. Thesis, Department of Mechanical Engineering, University of Waterloo, 1983. 